\title{
Research on the Vector Control for Electromechanical Integrated Toroidal Drive
}

\author{
Xin LIU, Lin ZHANG \\ Tianjin Key Laboratory of Modern Electromechanical Equipment Technology \\ Tianjin Polytechnic University, Tianjin, 300387, China \\ email: liuxin@tjpu.edu.cn
}

\section{Keywords: Vector Control; Electromechanical Integrated; Toroidal}

\begin{abstract}
Electromechanical integrated toroidal drive is a new-style composite drive, it integrates power and drive function. The mathematic models in coordinate system of the drive were derived based on the operating principle of the drive. In order to improve the controllability of toroidal drive, a vector control system with matrix converter and modulation was presented, and the corresponding simulation model was built. The response of speed, torque and currents in vector control system was simulated. The results justify the better controllability with vector control system. The research is useful for further practical application of the novel drive.
\end{abstract}

\section{Introduction}

Toroidal drive which is a kind of generalized composite drive can transmit large torque in a small size, and it is suitable for the engineering fields that require compactness, such as aviation and space flight, etc[1][2]. As more and more electromagnetic and electronic control techniques are utilized in mechanical engineering field, the generalized composite drives with integrated structure are still very limited. The electromagnetic harmonic drive [3] is an active drive in which the meshing forces between flexible gear and rigid one are controlled by electromagnetic force. In view of this, the electromechanical integrated toroidal drive as a kind of active composite drive without wear was presented, it integrates drive and power as a whole. The demand of electrical power is increasing enormously throughout the industry due to its easier controllability. Thus the electromechanical integrated toroidal drive with its speciality of integration has more expansive application prospect.

The new composite drive consists of four basic elements as shown in Figure 1: (a) the central worm 1; (b) radically positioned planets 2; (c) a toroidal shaped stator 3; and (d) a rotor 4, which forms the central output shaft upon which the planets are mounted. Among them, the central worm is fixed and the armature coils are mounted in helical grooves of its surface. The planets have permanent magnets instead of teeth. The $\mathrm{N}$ and $\mathrm{S}$ polar permanent magnets are mounted alternately on each planet. And the stator has several helical permanent magnets instead of helical teeth. In the same manner as planets, The $\mathrm{N}$ and $\mathrm{S}$ polar helical permanent magnets are mounted alternately on

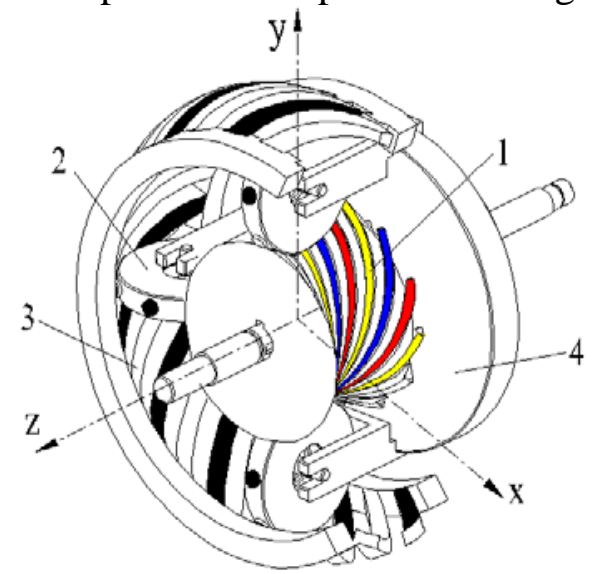

Fig.1 Diagram of electromechanical integrated toroidal drive the stator. When certain parameter relationship is satisfied between the planet pitch, helical angle of 
the stator and the pole-pairs number of central worm, the $\mathrm{N}$ pole of one element will correspond to $\mathrm{S}$ pole of the other elements. The electromagnetic force between $\mathrm{N}$ and $\mathrm{S}$ pole of the different elements are driving forces. A toroidal circular field is formed due to the alternate current in the coils of the worm. It drives the planets and the rotor rotate about their own axial. Thus, a power of low speed and large torque is output.

\section{Mathematic Models in Coordinate System}

Fig. 2 shows the relationship between coordinates at the cross section of the worm perpendicular to its axis at throat. The central worm is similar to the stator of the motor, and the revolution of the planets represents the movement of the rotor of the electromechanical integrated toroidal drive. $d$ and $q$ are direct and quadrature axes of the rotor. $i^{\mathrm{s}}$ is the resultant vector of three-phase current in worm armature.

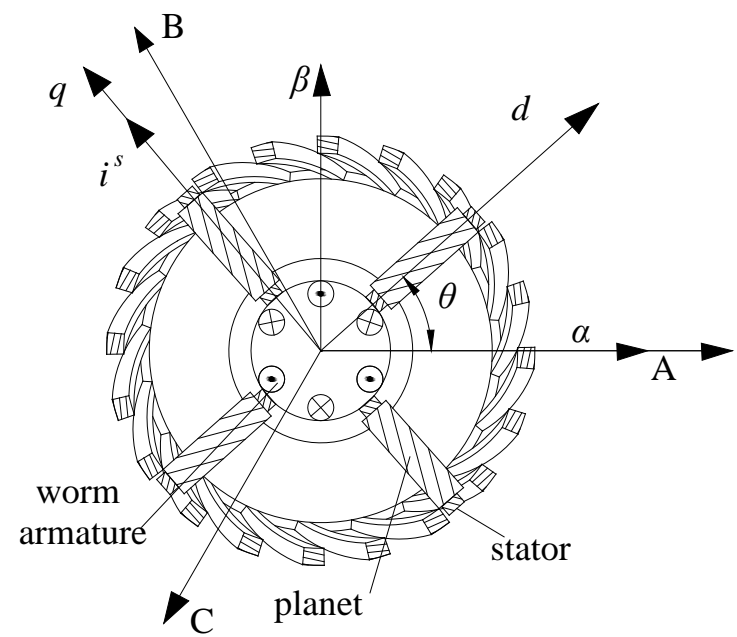

Fig.2 Relationship between coordinates of toroidal drive

The magnetic linkage of the worm armature caused by three-phase alternating current can be calculated as below:

$$
\left[\begin{array}{l}
\psi_{A} \\
\psi_{B} \\
\psi_{C}
\end{array}\right]=\left[\begin{array}{ccc}
L & M & M \\
M & L & M \\
M & M & L
\end{array}\right]\left[\begin{array}{l}
i_{A} \\
i_{B} \\
i_{B}
\end{array}\right]+\left[\begin{array}{c}
\psi_{r}^{A}(\theta) \\
\psi_{r}^{B}(\theta) \\
\psi_{r}^{C}(\theta)
\end{array}\right]
$$

Where $i_{A}, i_{B}$ and $i_{C}$ are the currents of the three phase coils, $\psi_{r}^{i}$ is the ith magnetic flux linkage caused by permanent magnets of planets on the rotor, $L$ is the self-induction of the magnetic field caused by the each phase coils, $M$ is the mutual inductance between the different phases, $\theta$ is the electrical angle between axis $d$ and phase A coil, $\theta=p_{n} \theta_{r}$, here $\theta_{r}$ is the mechanical angle of rotation of the rotor, $p_{n}$ is pole-pairs number of the rotor. Thus, the voltage balance equation of matrix form for the coils can be obtained:

$$
\left[\begin{array}{l}
u_{A} \\
u_{B} \\
u_{C}
\end{array}\right]=\left[\begin{array}{ccc}
R_{s} & 0 & 0 \\
0 & R_{s} & 0 \\
0 & 0 & R_{s}
\end{array}\right]\left[\begin{array}{l}
i_{A} \\
i_{B} \\
i_{C}
\end{array}\right]+p\left[\begin{array}{ccc}
L & M & M \\
M & L & M \\
M & M & L
\end{array}\right]\left[\begin{array}{l}
i_{A} \\
i_{B} \\
i_{B}
\end{array}\right]-\omega_{r} \psi_{f}\left[\begin{array}{c}
\sin \theta \\
\sin (\theta-2 \pi / 3) \\
\sin (\theta-4 \pi / 3)
\end{array}\right]
$$

Where $R_{S}$ is the resistance of each phase coils, $p=d / d t$ indicates the differential operation, $\psi_{f}$ is the equivalent amplitude of magnetic flux linkage for electromechanical integrated toroidal drive, $\omega_{r}$ is the equivalent electrical speed of the rotor. Due to the symmetry of the voltage balance equation, the diagonalizable form of voltage equation can be obtained. According to the relationship between coordinates in figure 2, the voltage balance equation in two-phase stationary coordinate can be given as: 


$$
\left[\begin{array}{l}
u_{\alpha} \\
u_{\beta}
\end{array}\right]=\left[\begin{array}{cc}
R_{\alpha}+L_{\alpha} \cdot p & 0 \\
0 & R_{\beta}+L_{\beta} \cdot p
\end{array}\right] \cdot\left[\begin{array}{c}
i_{\alpha} \\
i_{\beta}
\end{array}\right]+\omega_{r} \cdot \psi_{f \alpha \beta}\left[\begin{array}{c}
-\sin (\theta) \\
\cos (\theta)
\end{array}\right]
$$

Where $L_{\alpha}$ and $L_{\beta}$ are the inductances of the magnetic field caused by two phase coils in stationary coordinate, $L_{\alpha}=L_{\beta}=1.5(L-M), \psi_{f \alpha \beta}$ is the amplitude of magnetic flux linkage caused by two phase coils in stationary coordinate, $\psi_{f \alpha \beta}=\sqrt{3 / 2} \psi_{f}$.

The concept of vector control is to enable a motor to be controlled as if it were a dc motor[4][5]. Dynamic voltage equations in the stationary $\alpha \beta$ frame of the two-phase can be transformed into the $d q$ frame and vice versa by Park's transformation. The voltage balance equation in the two-phase rotating coordinate can be expressed as:

$$
\left[\begin{array}{l}
u_{d} \\
u_{q}
\end{array}\right]=\left[\begin{array}{cc}
R_{d}+L_{d} \cdot p & -L_{q} \omega_{e} \\
L_{d} \omega_{e} & R_{q}+L_{q} \cdot p
\end{array}\right] \cdot\left[\begin{array}{c}
i_{d} \\
i_{q}
\end{array}\right]+\omega_{r} \cdot \psi_{f d q}\left[\begin{array}{l}
0 \\
1
\end{array}\right]
$$

Where $\omega_{e}$ is electrical speed of the $d q$ frame, the current $i_{d}$ and $i_{q}$ are the equivalent direct currents at direct and quadrature axes, respectively. The angle of initial position between synchronous rotating coordinate and the axis of main pole for the worm of electromechanical integrated toroidal drive is zero. $k$ denotes the reduction ratio of the toroidal drive, for salient mounting permanent magnetic teeth of planets, $L_{q}$ is equal to $L_{q}$, thus, the developed torque of the rotor is expressed as:

$$
T=3 / 2 \cdot p_{n} \cdot k \psi_{f d q} i_{q}
$$

\section{Vector control for toroidal drive}

It is noticed that the rotor flux can be controlled by the stator current of worm armature in $d$ axis. Torque can be controlled by the stator current in $q$ axis. The speed command and reference currents in $d q$ frame are compared with their respective feedback values and regulated by PI controller. Closed-loop simulation model of the vector controller for electromechanical integrated toroial drive is shown in Fig. 3, in which the speed and current are both controlled.

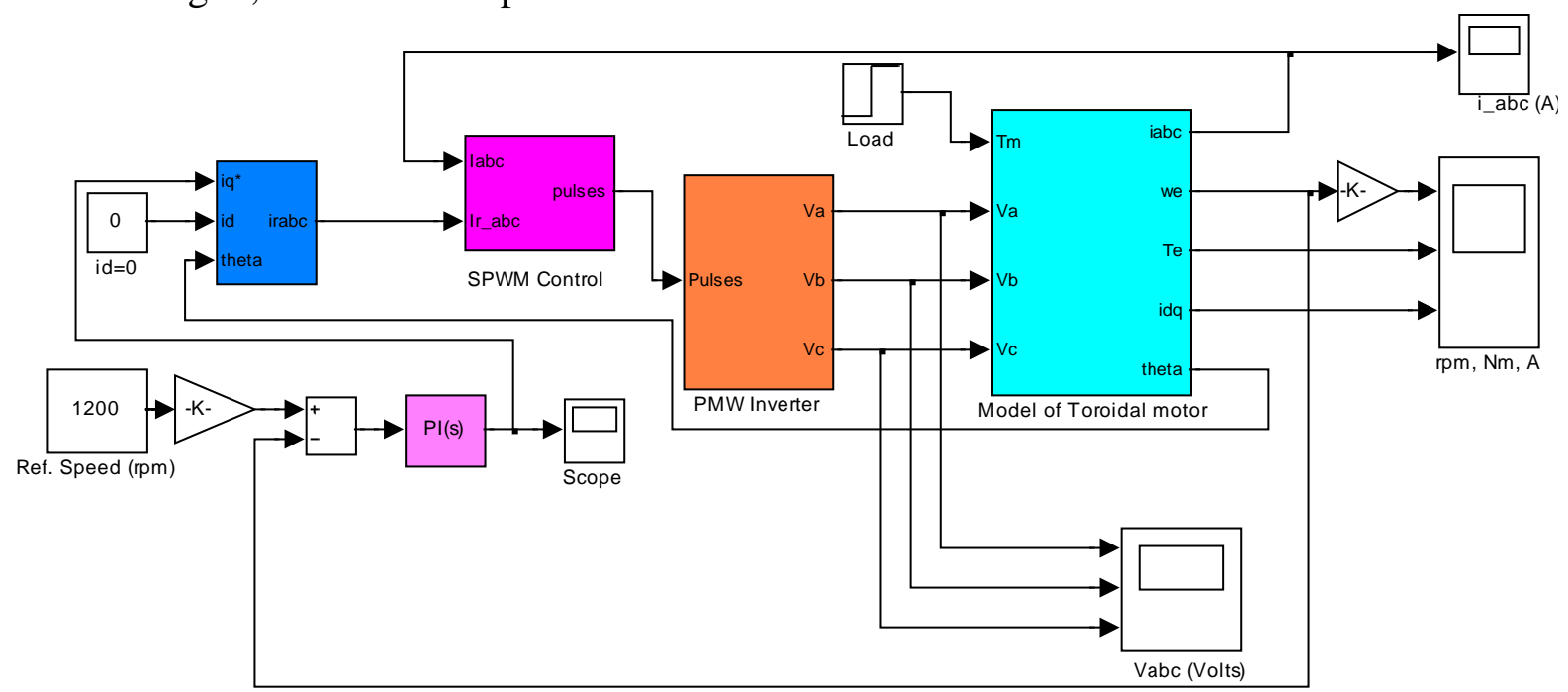

Fig.3 Simulation model of the vector control

In Fig.3, speed loop of the double closed-loop control system adopts PI control strategy. Gain parameters selection of PI controller is designed based on frequency response analysis. After the PI adjustment for difference between reference speed and speed feedback signal, the reference current in $q$ axis can be obtained. The current in $d$ axis is zero. A matrix converter is employed to control currents and rotor flux of the toroidal drive. Converted currents above require advanced modulation techniques, in this research, the space vector SPWM is adopted for the implementation. This modulation enables control of the input currents and the output voltages to be decoupled. The comparison between three-phase reference current and feedback current of the system controls PWM inverter, and the stator voltage of worm armature can be given. 


\section{Results and discusion}

Put the calculations parameters into the above simulation process, the simulation results of vector control are shown in Fig.4. Among them, the responses are the speed, torque and currents in $d q$ frame respectively.
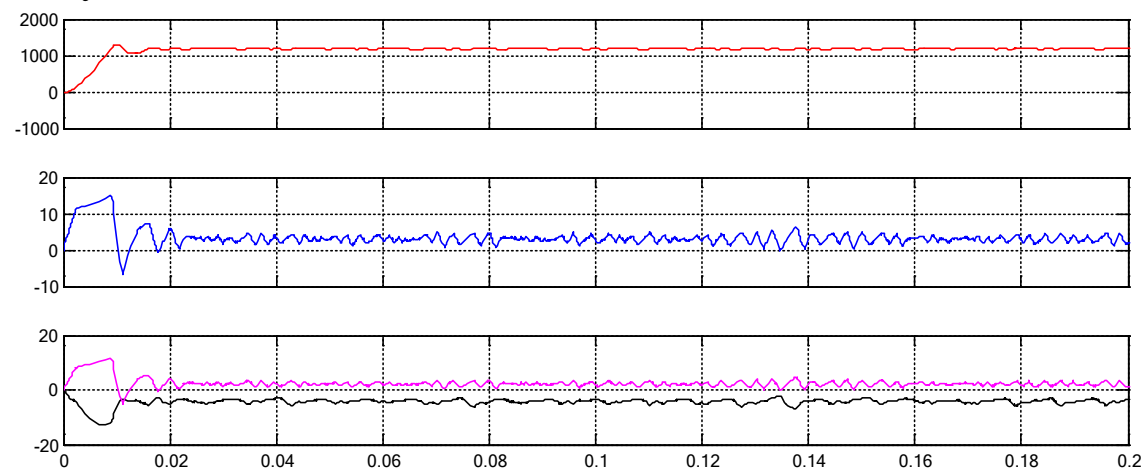

Fig.4 Simulation result of vector control

From the Fig.4, it is known that the overshoot of speed response at start time occurs. Meanwhile, larger torque fluctuation and the currents fluctuation occur. Soon after, the speed, the torque and the currents become stable state. Smaller fluctuations within stable torque and currents occur. It is caused by the periodic changes of the electromagnetic mesh between worm and planets within the electromechanical integrated toroidal drive system.

\section{Conclusion}

Based on the electromechanical integrated characteristic of toroidal drive system, the magnetic flux linkage equations for the toroidal worm armature are deduced. The voltage balance equation of the coils and the equation of the torque for the drive system are derived. The vector control system with matrix converter and modulation is presented, and the simulation model is built as well. The controllability of toroidal drive is realized. The responses of the main parameters in simulation result are analyzed. These research results are meaningful for further practical application of the novel drive.

\section{Acknowledgement}

The research in this paper was financially supported by the National Natural Science Foundation of China (No.51207107).and Innovation and Entrepreneurship Training Program of Tianjin polytechnic university (201410058125).

\section{References}

[1] Kuehnle,M.R., Peeken,H., and Troeder,C. The Toroidal drive[J]. Mechanical Engineering, 1981, 32(2):32-39.

[2] Lizhong Xu, Xiuhong Hao, Dynamic model of electromechanical integrated toroidal drive[J]. Applied Electromagnetics and Mechanics. 2005(22):199-211.

[3] Barth, Oliver. Harmonic piezodrive-miniaturized servomotor[J]. Mechatronics, 2000(10): 554-555.

[4] Ibraheem, Kumar P, Hasan N, Nizamuddin. Sub-optimal automatic generation control of interconnected power system using output vector feedback control strategy[J]. Electr Power Compon Syst 2012(40):977-994.

[5] S. Li, T.A. Haskew, Y. Hong, L. Xu, Direct-current vector control of three-phase grid-connected rectifier-inverter[J], Electr. Power Syst. 2011, 81 (2):357-366. 